International Journal of Pure and Applied Mathematics

Volume 104 No. 2 2015, 265-284

ISSN: 1311-8080 (printed version); ISSN: 1314-3395 (on-line version)

url: http://www.ijpam.eu

doi: http://dx.doi.org/10.12732/ijpam.v104i2.10

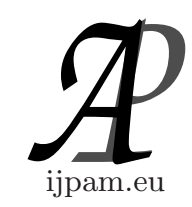

\title{
ON THE STABILITY OF TRIBONACCI AND $k$-TRIBONACCI FUNCTIONAL EQUATIONS IN MODULAR SPACE
}

\author{
Roji Lather ${ }^{1}$, Ashish $^{2}$, Manoj Kumar ${ }^{3}$ \\ ${ }^{1,3}$ Department of Mathematics \\ Maharshi Dayanand University \\ Rohtak, 124001, INDIA \\ ${ }^{2}$ Central University of Haryana \\ Jant-Pali, Mahendergarh, INDIA
}

Abstract: The purpose of this paper is to establish the Hyers-Ulam stability of the following Tribonacci and $k$-Tribonacci functional equations

$$
\begin{aligned}
f(x) & =f(x-1)+f(x-2)+f(x-3), \\
f(k, x) & =k f(k, x-1)+f(k, x-2)+f(k, x-3)
\end{aligned}
$$

in modular space.

AMS Subject Classification: 39B82, 39B52, 39B72

Key Words: Hyers-Ulam stability, modular space, Tribonacci functional equation, $k$-Tribonacci functional equation

\section{Introduction}

Stability is investigated when one is asking whether a small error of parameters in one problem causes a large deviation of its solution. Give an approximate homomorphism, is it possible to approximate it by a true homomorphism? In other words, we are looking for the situations when the homo-

Received: August 8, 2015

(c) 2015 Academic Publications, Ltd.

$\S$ Correspondence author url: www.acadpubl.eu 
morphisms are stable, that is, if a mapping is almost a homomorphism, then there exists a true homomorphism near it will small error as much as possible. This problem was posed by Ulam in 1940 [34] and is called the stability of functional equations. For Banach spaces, the problem was solved by Hyers [5] in the case of approximately additive mappings. Later, Hyers result was generalized by Aoki [35] for additive mappings and by Rassias [36] for linear mappings by allowing the Cauchy difference to be unbounded. During the last few decades, a number of papers and research monographs have been published on various generalizations and applications of generalized HyersUlam-Rassias stability to a number of functional equations and mappings (see $[2,3,4,6,7,8,9,10,11,13,15,18,19,20,24,25,26,27,28,29,30,31,33])$.

In 2009, S. M. Jung [32] investigated the Hyers-Ulam stability of Fibonacci functional equation. In 2011, Alvaro H. Salas [1] investigate about the $k$-Fibonacci number and their associated number. After that, M. Bidkhan and M. Hosslini [16] proved the stability of $k$-Fibonacci functional equation. Later on, M. Bidkhan et al. [17] succeeded to prove the Hyers-Ulam stability of $(k, s)$-Fibonacci functional equation. Furthermore, in 2012, M. Gordji, M. Naderi and Th. M. Rassias [22] et al. proved the stability of Tribonacci functional equation in nonArchimedean space and in 2014, M. E. Gordji, Ali Divandi, M. Rostannian, C. Park and D. Y. Sin [21] also proved the stability of Tribonacci functional equation in 2-normed space.

Recently, In 2014, M. N. Parizi et al. [23] and in 2015, Iz. El.-Fassi and S. Kabbaj [12] proved the stability of Fibonacci functional equation and orthogonal quadratic functional equation in Modular space respectively. In the first section of this paper, we denote by $T_{n}$ the $n$th Tribonacci number where

$$
T_{n}=T_{n-1}+T_{n-2}+T_{n-3} \quad \text { for } n=3
$$

with initial conditions $T_{0}=0, T_{1}=1, T_{2}=1$. From this, we may derive a functional equation

$$
f(x)=f(x-1)+f(x-2)+f(x-3)
$$

which is called the Tribonacci functional equation if a function $f: N \times R \rightarrow X$ satisfies the above equation for all $x \in R$. We denote the roots of equation $x^{3}-x^{2}-x-1=0$ by $p, q$ and $r$ where $q, r$ are complex, $|q|=|r|$ and $p$ is greater than one. We obtain

$$
p+q+r=1, \quad p q+q r+p r=-1, \quad p q r=1 .
$$


And in the second section, we denote by $F_{k, n}$ the $n$th $k$-Tribonacci number where

$$
F_{k, n}=k F_{k, n-1}+F_{k, n-2}+F_{k, n-3} \quad \text { for } n=3
$$

with initial conditions $F_{k, 0}=0, F_{k, 1}=1, F_{k, 2}=1$. From this, we may derive a functional equation

$$
f(k, x)=k f(k, x-1)+f(k, x-2)+f(k, x-3)
$$

which if called the $k$-Tribonacci function equation if a function $f: N \times R \rightarrow X$ satisfies the above equation for all $x \in R, K \in N$, characteristic equation of the $k$-Tribonacci sequence is $x^{3}-k x^{2}-x-1=0$, and $p, q, r$ denote the roots of characteristic equation where $p$ is greater than one and $q, r \in C$ and $|q|=|r|$. We know that $p+q+r=k, p q+q r+p r=-1, p q r=1$. For each $x \in R$, $[x]$ stands for the largest integer that does not exceed $x$. Finally, we prove the Hyers-Ulam stability of functional equations (3.1) and (3.2) respectively in modular space.

\section{Preliminaries}

In this section, we recall some definitions, basic notions and facts about Modular space. As, we know $p+q+r=1, p q+q r+p r=-1$ and $p q r=1$.

Now it follows from that

$$
\begin{aligned}
& f(x)-p(f(x-1)-r f(x-2))-r f(x-1) \\
& \quad=q[f(x-1)-(r+p) f(x-2)+\operatorname{pr} f(x-3)]
\end{aligned}
$$

for all $x=0$. By mathematical induction, we verify that for all $x=0$ and all $\mathrm{m}$ belonging to the set $\{0,1,2, \ldots\}$, we obtain,

$$
\begin{aligned}
& f(x)-p(f(x-1)-r f(x-2))-r f(x-1) \\
& \quad=q^{m}[f(x-m)-r f(x-m-1)+p r f(x-m-2)-p f(x-m-1)] \\
& f(x)-r[f(x-1)-q f(x-2)]-q f(x-1) \\
& \quad=p^{m}[f(x-m)-r f(x-m-1)+q r f(x-m-2)-q f(x-m-1)] \\
& f(x)-q[f(x-1)-p f(x-2)]-p f(x-1) \\
& \quad=r^{m}[f(x-m)-p f(x-m-1)+q p f(x-m-2)-q f(x-m-1)]
\end{aligned}
$$

for all $x=0$ and all $m \in\{0,1,2, \ldots\}$.

And in the similar way we can define for equation . 
Definition 2.1 ([12]). Let $X$ be an arbitrary vector space.

(a) A functional $\rho: X \rightarrow[0, \infty]$ is called a modular if for arbitrary $x, y \in X$,

(i) $\rho(x)=0$ if and only if $x=0$,

(ii) $\rho(\alpha x)=\rho(x)$ for every scalar $\alpha$ with $|\alpha|=1$,

(iii) $\rho(\alpha x+\beta y)=\rho(x)+\rho(y)$ if and only if $\alpha+\beta=1$ and $\alpha, \beta=0$,

(b) if (iii) is replaced by

(iii) $\rho(\alpha x+\beta y)=\alpha \rho(x)+\beta \rho(y)$ if and only if $\alpha+\beta=1$ and $\alpha, \beta=0$,

then we say that $\rho$ is a convex modular.

(c) A modular $\rho$ defines a corresponding modular space, i.e., the vector space $X_{\rho}$ given by

$$
X_{\rho}=\{x \in X: \rho(\lambda x) \rightarrow 0 \text { as } \lambda \rightarrow 0\}
$$

Definition $2.2([14])$. Let $\rho$ be a convex modular, the modular space $X_{\rho}$ can be equipped with a norm called the Luxemburg norm, defined by

$$
\|x\|_{\rho}=\inf \left\{\lambda>0: \rho \frac{x}{\lambda} \leq 1\right\}
$$

A function modular is said to be satisfy the $\Delta_{2}$-condition if there exit $k>0$ such that $\rho(2 x) \leq k \rho(x)$ for all $x \in X_{\rho}$.

Example 2.3 ([23]). Let $(X,\|\cdot\|)$ be a norm space, then $\|\cdot\|$ is a convex modular on $X$. But converse is not true.

In general the modular $\rho$ does not behave as a norm or as a distance because it is not sub-additive. But one can associate to a modular the F-norm (see [4]).

Definition $2.4([12])$. Let $\left\{x_{n}\right\}$ and $x$ be in $X_{\rho}$. Then

(i) we say $\left\{x_{n}\right\}$ is a $\rho$-convegent to $x$ and write $x_{n} \rho x$ if and only if $\rho\left(x_{n}-x\right) \rightarrow$ 0 as $n \rightarrow 0$,

(ii) the sequence $\left\{x_{n}\right\}$, with $x_{n} \rightarrow X_{\rho}$, is called $\rho$-Cauchy if $\rho\left(x_{n}-x_{m}\right) \rightarrow 0$ as $m, n \rightarrow \infty$,

(iii) a subset $S$ of $X_{\rho}$ is called $\rho$-complete if and only if any $\rho$-Cauchy sequence is $\rho$-convergent to an element of $S$. 
The modular $\rho$ has the Fatou property if and only if any $\rho(x) \leq \lim _{n \rightarrow \infty} \inf \rho\left(x_{n}\right)$ whenever the sequence $\left\{x_{n}\right\}$ is $\rho$-convergent to $x$. For further details and proofs, we refer the reader to [14].

Remark 2.5 ([12]). If $x \in X_{\rho}$ then $\rho(a x)$ is a nondecreasing function of $a \geq 0$. Suppose that $0 a b$, then property (iii) of Definition 2.1 with $y=0$ shows that

$$
\rho(a x)=\rho\left(\frac{a}{b} b x\right) \leq \rho(b x)
$$

Moreover, if $\rho$ is convex modular on $X$ and $|\alpha| \leq 1$ then, $\rho(\alpha x) \leq|\alpha| \rho(x)$ and also $\rho(x) \leq \frac{1}{2} \rho(2 x) \leq \frac{k}{2} \rho(x)$ if $\rho$ satisfy the $\Delta_{2}$-condition for all $x \in X$.

\section{Main Results}

\subsection{Stability of Tribonacci Functional Equation in Modular Space}

In the following theorem, we prove the Hyers-Ulam stability of the Tribonacci functional equation .

Theorem 3.1. Let $(X, \rho)$ be a Banach Modular space. If a function $f: R \rightarrow X$ satisfies the inequality

$$
\rho(f(x)-f(x-1)-f(x-2)-f(x-2))=\epsilon
$$

for all $x \in R$, and for some $\epsilon>0$, then there exist a Tribonacci function $H: N \times R \rightarrow X$ such that

$$
\rho(f(x)-H(x)) \leq \frac{2(1+|q|)+|q|^{2}}{\| q^{2}(r-p)+r^{2}(p-q)+p^{2}(q-r)} \times \frac{\epsilon}{1-|q|^{2}} .
$$

Proof. It follows from that

$$
\begin{aligned}
& \rho(f(x)-p\{f(x-1)-r f(x-2)\}-r f(x-1) \\
& \quad-q[f(x-1)-(r+p) f(x-2)+\operatorname{pr} f(x-3)]) \leq \epsilon
\end{aligned}
$$

If we replace $x$ by $x-\alpha$ in the last inequality, then we get

$$
\begin{aligned}
& \rho(f(x-\alpha)-p\{f(x-\alpha-1)-r f(x-\alpha-2)\}-r f(x-\alpha-1) \\
& \quad-q p f(x-\alpha-1)-(r+p) f(x-\alpha-2)+\operatorname{pr} f(x-\alpha-3)) \leq \epsilon
\end{aligned}
$$


for all $x \in R$.

Now multiplying both sides by $q^{\alpha}$,

$$
\begin{aligned}
& \rho\left(q^{\alpha}\{f(x-\alpha)-p[f(x-\alpha-1)-r f(x-\alpha-2)]-r f(x-\alpha-1)\}\right. \\
& \left.-q^{\alpha+1}\{f(x-\alpha-1)-(r+p) f(x-\alpha-2)+\operatorname{pr} f(x-\alpha-3)\}\right) \\
& \leq\left|q^{\alpha}\right| \rho(\{f(x-\alpha)-p[f(x-\alpha-1)-r f(x-\alpha-2)]-r f(x-\alpha-1)\} \\
& \left.\quad-q^{\alpha+1}\{f(x-\alpha-1)-(r+p) f(x-\alpha-2)+\operatorname{pr} f(x-\alpha-3)\}\right) \\
& \leq\left|q^{\alpha}\right| \epsilon
\end{aligned}
$$

for all $x \in R$ and $\alpha \in N$. Furthermore, we have

$$
\begin{aligned}
& \rho(\{f(x)-p[f(x-1)-r f(x-2)]-r f(x-1)\} \\
& \left.-q^{m}[f(x-m)-(r+p) f(x-m-1)+p r f(x-m-2)]\right) \\
& \leq \rho\left(\sum _ { \alpha = 0 } ^ { n - 1 } \left(q^{\alpha}[f(x-\alpha)-p\{f(x-\alpha-1)-r f(x-\alpha-2)\}-r f(x-\alpha-1)]\right.\right. \\
& \left.\left.\quad-q^{\alpha+1}[f(x-\alpha-1)-(r+p) f(x-\alpha-2)+p r f(x-\alpha-3)]\right)\right) \\
& \leq \sum_{\alpha=0}^{m-1}|q|^{\alpha}(\rho([f(x-\alpha)-p\{f(x-\alpha-1)-r f(x-\alpha-2)\}-r f(x-\alpha-1)] \\
& \quad-q[f(x-\alpha-1)-(r+p) f(x-\alpha-2)+p r f(x-\alpha-3)])) \\
& \leq \sum_{\alpha=0}^{m-1}|q|^{\alpha} \\
& \leq \frac{\epsilon}{1-|q|}
\end{aligned}
$$

for all $x \in R, m \in N$.

Let $x \in R$ be fixed, than implies that $\left\{q^{m}[f(x-m)-p(f(x-m-1)-\right.$ $r f(x-m-2))-r f(x-m-1))]$ is a cauchy sequence $(|q|<1)$. So by the completeness of $X$, we may define a function $H_{1}: R \rightarrow X$ such that

$$
\begin{array}{r}
H_{1}(x)=\lim _{m \rightarrow \infty} q^{m}[f(x-m)-(p+r) f(x-m-1)+p r f(x-m-2)] \\
\text { for all } x \in R .
\end{array}
$$

Applying the definition of $H_{1}$, we introduce the Tribonacci function

$$
H_{1}(x-1)+H_{1}(x-2)+H_{1}(x-3)
$$




$$
\begin{aligned}
= & q^{-1} \lim _{m \rightarrow \infty} q^{m+1}[f(x-(m+1))-(p+r) f(x-(m+1)-1)+\operatorname{pr} f(x-(m+1)-2)] \\
& +q^{-2} \lim _{m \rightarrow \infty} q^{m+2}[f(x-(m+2))-(p+r) f(x-(m+2)-1)+\operatorname{pr} f(x-(m+2)-2)] \\
& +q^{-3} \lim _{m \rightarrow \infty} q^{m+3}[f(x-(m+3))-(p+r) f(x-(m+3)-1)+\operatorname{pr} f(x-(m+3)-2)] \\
= & q^{-1} H_{1}(x)+q^{-2} H_{1}(x)+q^{-3} H_{1}(x) \\
= & H_{1}(x) \quad \text { for all } x \in R .
\end{aligned}
$$

Hence $H_{1}$ is a Tribonacci function.

If $m \rightarrow \infty$, then from, we obtain

$$
\rho\left(f(x)-(p+r) f(x-1)+p r f(x-2)-H_{1}\right)=\frac{1}{1-|q|} \epsilon .
$$

for all $x \in R$. Furthermore, it follows from that

$$
\begin{aligned}
& \rho([f(x)-q\{f(x-1)-p f(x-2)\}-p f(x-1)] \\
& \quad-r[f(x-1)-p f(x-2)+p q f(x-3)-q f(x-2)])=\epsilon
\end{aligned}
$$

for all $x \in R$. Now, we replace $x$ by $x-\alpha$ in above inequality, we have

$$
\begin{aligned}
& \rho([f(x-\alpha)-q(f(x-\alpha-1)-p f(x-\alpha-2))-p f(x-\alpha-1)] \\
& \quad-r[f(x-\alpha-1)-p f(x-\alpha-2)+p q f(x-\alpha-3)-q f(x-\alpha-2)])=\epsilon
\end{aligned}
$$

and now multiplying by $r^{\alpha}$ on both sides.

$$
\begin{aligned}
& \rho\left(r^{\alpha}[f(x-\alpha)-q\{f(x-\alpha-1)-p f(x-\alpha-2)\}-p f(x-\alpha-1)]\right. \\
& \left.\quad-r^{\alpha+1}[f(x-\alpha-1)-p f(x-\alpha-2)+p q f(x-\alpha-3)-q f(x-\alpha-2)]\right) \\
& \leq\left|r^{\alpha}\right|(\rho([f(x-\alpha)-q\{f(x-\alpha-1)-p f(x-\alpha-2)\}-p f(x-\alpha-1)] \\
& \left.\quad-r^{\alpha+1}[f(x-\alpha-1)-p f(x-\alpha-2)+p q f(x-\alpha-3)-q f(x-\alpha-2)]\right) \\
& \leq\left|r^{\alpha}\right| \epsilon
\end{aligned}
$$

for all $x \in R, \alpha \in Z$. Now, we have

$$
\begin{aligned}
& \rho([f(x)-q\{f(x-1)-p f(x-2)\}-p f(x-1)] \\
& \quad-r^{m}[f(x-m)-(q+p) f(x-m-1)+p q(f(x-m-2)]) \\
& \leq \rho\left(\sum _ { k = 1 } ^ { m } \left(r^{\alpha}[f(x-\alpha)-q\{f(x-\alpha-1)-p f(x-\alpha-2)\}-p f(x-\alpha-1)]\right.\right.
\end{aligned}
$$




$$
\begin{aligned}
& \left.\left.-r^{\alpha+1}[f(x-\alpha-1)-(p+q) f(x-\alpha-2)+p q f(x-\alpha-3)]\right)\right) \\
\leq & \sum_{k=1}^{m}|r|^{\alpha}(\rho([f(x-\alpha)-q\{f(x-\alpha-1)-p f(x-\alpha-2)\}-p f(x-\alpha-1)] \\
& \quad-r[f(x-\alpha-1)-(p+q) f(x-\alpha-2)+p q f(x-\alpha-3)])) \\
\leq & \sum_{k=1}^{m}|r|^{\alpha} \\
\leq & \frac{\epsilon}{1-|r|}
\end{aligned}
$$

for all $x \in R$ and $m \in N$. We have

$$
\left\{r^{m}[f(x-m)-(q+p) f(x-m-1)+p q f(x-m-2)]\right\}
$$

is a cauchy sequence $(|r|<1)$ for all $x \in R$. Hence, we can define a function $H_{2}: R \rightarrow X$ by

$$
H_{2}(x)=\lim _{m \rightarrow \infty} r^{m}[f(x-m)-(q+p) f(x-m-1)+p q f(x-m-2)]
$$

for all $x \in R$. Using the above definition of $H_{2}$, we have

$$
\begin{aligned}
H_{2}(x-1)+H_{2}(x-2)+H_{2}(x-3) \\
\left.=r^{-1} \lim _{m \rightarrow \infty} r^{m+1}[f(x)-(m+1))-(q+p) f(x-(m+1)-1)+p q f(x-(m+1)-2)\right] \\
\quad+r^{-2} \lim _{m \rightarrow \infty} r^{m+2}[f(x-(m+2))-(q+p) f(x-(m+2)-1)+p q f(x-(m+2)-2)] \\
\quad+r^{-3} \lim _{m \rightarrow \infty} r^{m+3}[f(x-(m+3))-(q+p) f(x-(m+3)-1)+p q f(x-(m+3)-2)] \\
=r^{-1} H_{2}(x)+r^{-2} H_{2}(x)+r^{-3} H_{2}(x) \\
=H_{2}(x) \quad \text { for all } x \in R .
\end{aligned}
$$

So, we can say that $H_{2}$ is also a Tribonacci function. If $m$ tends to $\infty$, then from, we have

$$
\begin{aligned}
\rho\left(f(x)-(q+p) f(x-1)+q p f(x-2)-H_{2}(x)\right) & =\frac{1}{1-|r|} \epsilon \\
& =\frac{1}{1-|q|} \epsilon .
\end{aligned}
$$

for all $x \in R$. Finally, analogous to, we obtain

$$
\rho([f(x)-r\{f(x-1)-q f(x-2)\}-q f(x-1)]
$$




$$
-p[f(x-1)-r f(x-2)+q r f(x-3)-q f(x-2)]) \leq \epsilon
$$

for all $x \in R$.

Now we replace $x$ by $x+\alpha$ in above inequality, that we have

$$
\begin{aligned}
& \rho(f(x+\alpha)-r\{f(x+\alpha-1)-q f(x+\alpha-2)\}-q f(x+\alpha-1) \\
& \quad-p[f(x+\alpha-1)-(r+q) f(x-\alpha-2)+q r f(x+\alpha-3)]) \leq \epsilon
\end{aligned}
$$

and

$$
\begin{aligned}
& \rho\left(p^{-\alpha}[f(x+\alpha)-r\{f(x+\alpha-1)-q f(x+\alpha-2)\}-q f(x+\alpha-1)]\right. \\
& \left.\left.-p^{-\alpha+1}[f(x+\alpha-1)-(r+q) f(x-\alpha-2))+q r f(x+\alpha-3)\right]\right) \\
& \quad \leq\left|\alpha^{-1}\right|^{k} \epsilon
\end{aligned}
$$

for all $x \in R$ and $\alpha \in Z$. Applying, we obtain that

$$
\begin{gathered}
\rho\left(p^{-m}[f(x+m)-r\{f(x+m-1)-q f(x+m-2)\}\right. \\
-q f(x+m-1)]-[f(x)-(r+q) f(x-1)+r q f(x-2)]) \\
\leq \sum_{\alpha=1}^{m} \rho\left(p^{-\alpha}[f(x+\alpha)-r\{f(x+\alpha-1)-q f(x+\alpha-2)\}-q f(x+\alpha-1)]\right. \\
\left.\quad-p^{-\alpha+1}[f(x+\alpha-1)-(r+q) f(x+\alpha-2)+q r f(x+\alpha-3)]\right) \\
\leq \sum_{\alpha=1}^{m} p^{-\alpha}(\rho([f(x+\alpha)-r\{f(x+\alpha-1)-q f(x+\alpha-2)\}-q f(x+\alpha-1)] \\
\quad-p[f(x+\alpha-1)-(r+q) f(x+\alpha-2)+q r f(x+\alpha-3)])) \\
\leq \sum_{\alpha=1}^{m} p^{-\alpha} \epsilon
\end{gathered}
$$

for all $x \in R, m \in N$. We obviously have

$$
\left\{p^{-m}[f(x+m)-(r+q) f(x+m-1)+q r f(x+m-2)]\right\}
$$

is a cauchy sequence by definition of completeness for a fixed $x \in R$. Hence, we may define a function $H_{3}: R \rightarrow X$ by

$$
H_{3}(x)=\lim _{m \rightarrow \infty} p^{-m}[f(x+m)-(r+q) f(x+m+1)+q r f(x+m-2)]
$$


for all $x \in R$. In view of above definition of $H_{3}$, we obtain

$$
\begin{aligned}
H_{3}(x-1)+H_{3}(x-2)+H_{3}(x-3) \\
=p^{-1} \lim _{m \rightarrow \infty} p^{-(m-1)}[f(x+m-1)-(r+q) f(x+(m-1)-1)+q r f(x+(m-1)-2)] \\
\quad+p^{-2} \lim _{m \rightarrow \infty} p^{-(m-2)}[f(x+m-2)-(r+q) f(x+(m-2)-1)+q r f(x+(m-2)-2)] \\
\quad+p^{-3} \lim _{m \rightarrow \infty} p^{-(m-3)}[f(x+m-3)-(r+q) f(x+(m-3)-1)+q r f(x+(m-3)-2)] \\
=p^{-1} H_{3}(x)+p^{-2} H_{3}(x)+p^{-3} H_{3}(x) \\
=H_{3}(x) \quad \text { for all } x \in R .
\end{aligned}
$$

Hence, we can say that $H_{3}$ is also a Tribonacci function. If we suppose, $m$ tends to infinity in then we have

$$
\rho\left(H_{3}(x)-f(x)+(r+q) f(x-1)-q r f(x-2)\right)=\frac{\alpha^{-1}}{1-\left|\alpha^{-1}\right|} \epsilon
$$

for all $x \in R$. From, and, we observe that

$$
\begin{aligned}
& \rho\left(f(x)-\left[\frac{q^{2}(r-p) H_{1}(x)+r^{2}(p-q) H_{2}(x)-p^{2}(q-r) H_{3}(x)}{q^{2}(r-p)+r^{2}(p-q)+p^{2}(q-r)}\right]\right) \\
& =\frac{1}{\left|q^{2}(r-p)+r^{2}(p-q)+p^{2}(q-r)\right|} \rho\left(\left\{q^{2}(r-p)+r^{2}(p-q)+p^{2}(q-r)\right\}\right. \\
& \left.\quad \times f(x)-q^{2}(r-p) H_{1}(x)-r^{2}(p-q) H_{2}(x)+p^{2}(q-r) H_{3}(x)\right)
\end{aligned}
$$

Now, we assume that

$$
\begin{aligned}
& \quad \frac{1}{\left|q^{2}(r-p)+r^{2}(p-q)+p^{2}(q-r)\right|}=\frac{1}{|A|} \\
& \leq \frac{1}{|A|} \rho\left(\left[q^{2}(r-p) f(x)-q^{2}\left(r^{2}-p^{2}\right) f(x-1)+q^{2}(r-p) p r f(x-2)-q^{2}(r-p) H_{1}(x)\right]\right. \\
& \quad+\left[r^{2}(p-q) f(x)-r^{2}\left(p^{2}-q^{2}\right) f(x-1)+r^{2}(p-q) q p f(x-2)-r^{2}(p-q) H_{2}(x)\right] \\
& \left.\quad+\left[p^{2}(q-r) f(x)-p^{2}\left(q^{2}-r^{2}\right) f(x-1)+p^{2}(q-r) q r f(x-2)-p^{2}(q-r) H_{3}(x)\right]\right) \\
& \leq \frac{1}{|A|}\left[\frac{1}{1-|q|}+\frac{1}{1-|q|}+\frac{\left|q^{2}\right|}{1-\left|q^{2}\right|}\right] \epsilon \\
& =\frac{1}{|A|}\left[\frac{2}{1-|q|}+\frac{\left|q^{2}\right|}{1-\left|q^{2}\right|}\right] \epsilon \\
& =\frac{1}{|A|}\left[\frac{2(1+|q|)+|q|^{2}}{1-\left|q^{2}\right|}\right] \epsilon
\end{aligned}
$$


Putting the value of $|A|$ from we get the required result.

Hence,

$$
H(x)=\frac{q^{2}(r-p) H_{1}(x)+r^{2}(p-q) H_{2}(x)-p^{2}(q-r) H_{3}(x)}{q^{2}(r-p)+r^{2}(p-q)+p^{2}(q-r)}
$$

for all $x \in R$. It is not difficult to show that $H$ is a Tribonacci function satisfying

\subsection{Stability of $k$-Tribonacci Functional Equation in Modular Space}

Throughout the following theorem, we prove the Hyers-Ulam stability of the $k$-Tribonacci functional equation .

Theorem 3.2. Let $(X, \rho)$ be a Banach modular space. If a function $f$ : $R \rightarrow X$ satisfies the inequality

$$
\rho(f(k, x)-k f(k, x-1)-f(k, x-2)-f(k, x-2))=\epsilon
$$

for all $x \in R, k \in N$ and for some $\epsilon>0$, then there exist a $k$-Tribonacci function $H: N \times R \rightarrow X$ such that

$$
\begin{aligned}
& \rho(f(k, x)-H(k, x)) \\
& \quad=\frac{2(1+|q|)+|q|^{2}}{\| q^{2}(r-p)+r^{2}(p-q)+p^{2}(q-r)} \times \frac{\epsilon}{1-|q|^{2}} .
\end{aligned}
$$

Proof. Since, $p+q+r=k, p q+q r+p r=-1$ and $p q r=1$. So from, we obtain

$$
\begin{aligned}
& \rho(f(k, x)-(p+q+r) f(k, x-1)+(p q+q r+p r) f(k, x-2) \\
& \quad-\operatorname{pqr} f(k, x-3))=\epsilon .
\end{aligned}
$$

for all $x \in R, k \in N$. Now it follows from that

$$
\begin{aligned}
& f(k, x)-p(f(k, x-1)-r f(k, x-2))-r f(k, x-1) \\
& \quad-q[f(k, x-1)-(r+p) f(k, x-2)+p r f(k, x-3)]=\epsilon
\end{aligned}
$$

for all $k \in N, x \geq 0$.

If we replace $x$ by $x-\alpha$ in inequality then we get

$$
\begin{aligned}
& \rho(f(k, x-\alpha)-p[f(k, x-\alpha-1)-r f(k, x-\alpha-2)] \\
& \quad-r f(k, x-\alpha-1)-q p f(K, x-\alpha-1)-(r+p) f(k, x-\alpha-2)
\end{aligned}
$$




$$
+\operatorname{pr} f(k, x-\alpha-3))=\epsilon
$$

for all $x \in R, k \in N$.

Now multiplying both sides by $q^{\alpha}$,

$$
\begin{aligned}
& \rho\left(q^{\alpha}[f(k, x-\alpha)-p\{f(k, x-\alpha-1)-r f(k, x-\alpha-2)\}-r f(k, x-\alpha-1)]\right. \\
& \left.-q^{\alpha+1}[f(k, x-\alpha-1)-(r+p) f(k, x-\alpha-2)+p r f(k, x-\alpha-3)]\right) \\
& \quad \leq\left|q^{\alpha}\right| \rho([f(k, x-\alpha)-p\{f(k, x-\alpha-1)-r f(k, x-\alpha-2)\}-r f(k, x-\alpha-1)] \\
& -q[f(k, x-\alpha-1)-(r+p) f(k, x-\alpha-2)+\operatorname{pr} f(k, x-\alpha-3)]) \\
& \quad \leq\left|q^{\alpha}\right| \epsilon
\end{aligned}
$$

for all $x \in R, k \in N$. Furthermore, we have

$$
\begin{aligned}
& \rho(f(k, x)-p\{f(k, x-1)-r f(k, x-2)\}-r f(k, x-1) \\
& \left.-q^{m}[f(k, x-m)-(r+p) f(k, x-m-1)+p r f(k, x-m-2)]\right) \\
\leq & \rho\left(\sum_{\alpha=0}^{m-1} q^{\alpha}[f(k, x-\alpha)-p\{f(k, x-\alpha-1)-r f(k, x-\alpha-2)\}-r f(k, x-\alpha-1)]\right. \\
& \left.-q^{\alpha+1}[f(k, x-\alpha-1)-(r+p) f(k, x-\alpha-2)+p r f(k, x-\alpha-3)]\right) \\
\leq & \sum_{\alpha=0}^{m-1}|q|^{\alpha} \rho([f(k, x-\alpha)-p\{f(k, x-\alpha-1)-r f(k, x-\alpha-2)\}-r f(k, x-\alpha-1)] \\
& -q[f(k, x-\alpha-1)-(r+p) f(k, x-\alpha-2)+p r f(k, x-\alpha-3)]) \\
\leq & \sum_{\alpha=0}^{m-1}|q|^{\alpha} \epsilon \\
\leq & \frac{\epsilon}{1-|q|}
\end{aligned}
$$

for all $x \in R, m \in N, k \in N$.

Let $x \in R$ be fixed, than implies that $\left\{q^{m}[f(k, x-m)-p(f(k, x-m-1)-\right.$ $r f(k, x-m-2))-r f(k, x-m-1))]$ is a cauchy sequence $(|q|<1)$. So by the completeness of $X$, we may define a function $H_{1}: R \rightarrow X$ such that

$$
\begin{aligned}
H_{1}(k, x)= & \lim _{m \rightarrow \infty} q^{m}[f(k, x-m)-(p+r) f(k, x-m-1) \\
& +\operatorname{prf}(k, x-m-2)] \quad \text { for all } x \in R, k \in N .
\end{aligned}
$$


Applying the definition of $H_{1}$, we introduce the $k$-Tribonacci function

$$
\begin{aligned}
& k H_{1}(k, x-1)+H_{1}(k, x-2)+H_{1}(k, x-3) \\
& =k q^{-1} \lim _{m \rightarrow \infty} q^{m+1}[f(k, x-(m+1)) \\
& -(p+r) f(k, x-(m+1)-1)+p r f(k, x-(m+1)-2)] \\
& +q^{-2} \lim _{m \rightarrow \infty} q^{m+2}[f(k, x-(m+2)) \\
& -(p+r) f(k, x-(m+2)-1)+p r f(k, x-(m+2)-2)] \\
& +q^{-3} \lim _{m \rightarrow \infty} q^{m+3}[f(k, x-(m+3)) \\
& -(p+r) f(k, x-(m+3)-1)+p r f(k, x-(m+3)-2)] \\
& =k q^{-1} H_{1}(k, x)+q^{-2} H_{1}(k, x)+q^{-3} H_{1}(k, x) \\
& =H_{1}(k, x) \quad \text { for all } x \in R, k \in N \text {. }
\end{aligned}
$$

Hence $H_{1}$ is a $k$-Tribonacci function.

If $m \rightarrow \infty$, then from we obtain

$$
\begin{aligned}
& \rho\left(f(k, x)-(p+r) f(k, x-1)+\operatorname{pr} f(k, x-2)-H_{1}(k, x)\right) \\
& \quad \leq \frac{1}{1-|q|} \epsilon
\end{aligned}
$$

for all $x \in R, k \in N$. Furthermore, it follows from that

$$
\begin{aligned}
& \rho(f(k, x)-q(f(k, x-1)-p f(k, x-2))-p f(k, x-1) \\
& \quad-r[f(k, x-1)-p f(k, x-2)+p q f(k, x-3)-q f(k, x-2)])=\epsilon
\end{aligned}
$$

for all $x \in R, k \in N$. Now, we replace $x$ by $x-\alpha$ in above inequality, we have

$$
\begin{aligned}
& \rho(f(k, x-\alpha)-q(f(k, x-\alpha-1)-p f(k, x-\alpha-2))-p f(k, x-\alpha-1) \\
& \quad-r[f(k, x-\alpha-1)-p f(k, x-\alpha-2)+p q f(k, x-\alpha-3)-q f(k, x-\alpha-2)])=\epsilon
\end{aligned}
$$

and now multiplying by $r^{\alpha}$ on both sides.

$$
\begin{aligned}
& \rho\left(r^{\alpha}[f(k, x-\alpha)-q(f(k, x-\alpha-1)-p f(k, x-\alpha-2))-p f(k, x-\alpha-1)]\right. \\
& \left.\quad-r^{\alpha+1}[f(K, x-\alpha-1)-p f(K, x-\alpha-2)+p q f(k, x-\alpha-3)-q f(k, x-\alpha-2)]\right) \\
& \leq\left|r^{\alpha}\right| \rho([f(k, x-\alpha)-q(f(k, x-\alpha-1)-p f(k, x-\alpha-2))-p f(k, x-\alpha-1)] \\
& \quad-r[f(K, x-\alpha-1)-p f(K, x-\alpha-2)+p q f(k, x-\alpha-3)-q f(k, x-\alpha-2)]) \\
& \leq\left|r^{\alpha}\right| \epsilon
\end{aligned}
$$


for all $x \in R, \alpha \in Z$. Now, we have

$$
\begin{aligned}
\rho( & f(k, x)-q\{f(k, x-1)-p f(k, x-2)\}-p f(k, x-1) \\
& -r^{m}[f(k, x-m)-(q+p) f(k, x-m-1)+p q(f(k, x-m-2)]) \\
\leq & \rho\left(\sum_{\alpha=0}^{m-1} r^{\alpha}[f(k, x-\alpha)-q\{f(k, x-\alpha-1)-p f(k, x-\alpha-2)\}-p f(k, x-\alpha-1)]\right. \\
& \left.-r^{\alpha+1}[f(k, x-\alpha-1)-(p+q) f(k, x-\alpha-2)+p q f(k, x-\alpha-3)]\right) \\
\leq & \sum_{\alpha=0}^{m-1}|r|^{\alpha} \rho([f(k, x-\alpha)-q\{f(k, x-\alpha-1)-p f(k, x-\alpha-2)\}-p f(k, x-\alpha-1)] \\
& -r[f(k, x-\alpha-1)-(p+q) f(k, x-\alpha-2)+p q f(k, x-\alpha-3)]) \\
\leq & \sum_{\alpha=0}^{m-1}|r|^{\alpha} \epsilon \\
\leq & \frac{\epsilon}{1-|r|}
\end{aligned}
$$

for all $x \in R$ and $m \in N$. We have

$$
\left\{r^{m}[f(k, x-m)-(q+p) f(k, x-m-1)+p q f(k, x-m-2)]\right\}
$$

is a cauchy sequence $(|r|<1)$ for all $x \in R$. Hence, we can define a function $H_{2}: R \rightarrow X$ by

$$
\begin{aligned}
& H_{2}(k, x) \\
& =\lim _{m \rightarrow \infty} r^{m}[f(k, x-m)-(q+p) f(k, x-m-1)+p q f(k, x-m-2)]
\end{aligned}
$$

for all $x \in R$. Using the above definition of $H_{2}$, we have

$$
\begin{aligned}
& k H_{2}(k, x-1)+H_{2}(k, x-2)+H_{2}(k, x-3) \\
& \begin{aligned}
k & r^{-1} \lim _{m \rightarrow \infty} r^{m+1}[f(k, x-(m+1)) \\
& \quad-(q+p) f(k, x-(m+1)-1)+p q f(k, x-(m+1)-2)] \\
& +r^{-2} \lim _{m \rightarrow \infty} r^{m+2}[f(k, x-(m+2)) \\
& \quad-(q+p) f(k, x-(m+2)-1)+p q f(k, x-(m+2)-2)] \\
& +r^{-3} \lim _{m \rightarrow \infty} r^{m+3}[f(k, x-(m+3))
\end{aligned}
\end{aligned}
$$




$$
\begin{aligned}
& -(q+p) f(k, x-(m+3)-1)+p q f(k, x-(m+3)-2)] \\
= & k r^{-1} H_{2}(k, x)+r^{-2} H_{2}(k, x)+r^{-3} H_{2}(k, x) \\
= & H_{2}(k, x) \quad \text { for all } x \in R .
\end{aligned}
$$

So, we can say that $H_{2}$ is also a $k$-Tribonacci function. If $m$ tends to $\infty$, then from, we have

$$
\begin{aligned}
& \rho\left(f(k, x)-(q+p) f(k, x-1)+q p f(k, x-2)-H_{2}(k, x)\right) \\
& \quad \leq \frac{1}{1-|r|} \epsilon \leq \frac{1}{1-|q|} \epsilon .
\end{aligned}
$$

for all $x \in R$. Finally, analogous to , we obtain

$$
\begin{array}{r}
\rho(f(k, x)-r\{f(k, x-1)-q f(k, x-2)\}-q f(k, x-1) \\
-p[f(k, x-1)-r f(k, x-2)+q r f(k, x-3)-q f(k, x-2)])=\epsilon
\end{array}
$$

for all $x \in R$. Now we replace $x$ by $x+\alpha$ in above inequality, that we have

$$
\begin{aligned}
& \rho(f(k, x+\alpha)-r\{f(k, x+\alpha-1)-q f(k, x+\alpha-2)\}-q f(k, x+\alpha-1) \\
& -p[f(k, x+\alpha-1)-(r+q) f(k, x-\alpha-2)+q r f(k, x+\alpha-3)]) \leq \epsilon
\end{aligned}
$$

and

$$
\begin{aligned}
& \rho\left(p^{-\alpha}[f(k, x+\alpha)-r(f(k, x+\alpha-1)-q f(k, x+\alpha-2))-q f(k, x+\alpha-1)]\right. \\
& \left.\left.-p^{-\alpha+1}[f(k, x+\alpha-1)-(r+q) f(k, x-\alpha-2))+q r f(k, x+\alpha-3)\right]\right) \\
& \quad=\left|\alpha^{-1}\right|^{k} \epsilon
\end{aligned}
$$

for all $x \in R$ and $\alpha \in Z$. Applying, we obtain that

$$
\begin{aligned}
& \rho\left(p^{-m}[f(k, x+m)-r(f(k, x+m-1)-q f(k, x+m-2))\right. \\
&-q f(k, x+m-1)]-[f(k, x)-(r+q) f(k, x-1)+r q f(k, x-2)]) \\
& \leq \sum_{\alpha=1}^{m} \rho\left(p^{-\alpha}[f(k, x+\alpha)-r(f(k, x+\alpha-1)-q f(k, x+\alpha-2))-q f(k, x+\alpha-1)]\right. \\
&\left.-p^{-\alpha+1}[f(k, x+\alpha-1)-(r+q) f(k, x+\alpha-2)+q r f(k, x+\alpha-3)]\right) \\
& \leq \sum_{\alpha=1}^{m} p^{-\alpha} \rho([f(k, x+\alpha)-r(f(k, x+\alpha-1)-q f(k, x+\alpha-2))-q f(k, x+\alpha-1)] \\
&\left.\quad-p^{-\alpha+1}[f(k, x+\alpha-1)-(r+q) f(k, x+\alpha-2)+q r f(k, x+\alpha-3)]\right)
\end{aligned}
$$




$$
\leq \sum_{\alpha=1}^{m} p^{-\alpha} \epsilon
$$

for all $x \in R, m \in N$. By using we see that

$$
\left\{p^{-m}[f(k, x+m)-(r+q) f(k, x+m-1)+q r f(k, x+m-2)]\right\}
$$

is a cauchy sequence by definition of completeness for a fixed $x \in R$. Hence, we may define a function $H_{3}: R \rightarrow X$ by

$$
H_{3}(k, x)=\lim _{m \rightarrow \infty} p^{-m}[f(k, x+m)-(r+q) f(k, x+m+1)+q r f(k, x+m-2)]
$$

for all $x \in R$. In view of above definition of $H_{3}$, we obtain

$$
\begin{aligned}
& k H_{3}(k, x-1)+H_{3}(k, x-2)+H_{3}(k, x-3) \\
& =k p^{-1} \lim _{m \rightarrow \infty} p^{-(m-1)}[f(k, x+m-1) \\
& -(r+q) f(k, x+(m-1)-1)+q r f(k, x+(m-1)-2)] \\
& +p^{-2} \lim _{m \rightarrow \infty} p^{-(m-2)}[f(k, x+m-2) \\
& -(r+q) f(k, x+(m-2)-1)+q r f(k, x+(m-2)-2)] \\
& +p^{-3} \lim _{m \rightarrow \infty} p^{-(m-3)}[f(k, x+m-3) \\
& -(r+q) f(k, x+(m-3)-1)+q r f(k, x+(m-3)-2)] \\
& =k p^{-1} H_{3}(k, x)+p^{-2} H_{3}(k, x)+p^{-3} H_{3}(k, x) \\
& =H_{3}(k, x) \text { for all } x \in R, k \in N \text {. }
\end{aligned}
$$

Hence, we can say that $H_{3}$ is also a $k$-Tribonacci function. If we suppose, $m$ tends to infinity in then we have

$$
\begin{aligned}
& \rho\left(H_{3}(k, x)-f(k, x)+(r+q) f(k, x-1)-q r f(k, x-2)\right) \\
& \quad \leq \frac{\alpha^{-1}}{1-\left|\alpha^{-1}\right|} \epsilon
\end{aligned}
$$

for all $x \in R$. From (22), (25) and (28), we observe that

$$
\begin{aligned}
& \rho\left(f(k, x)-\left[\frac{q^{2}(r-p) H_{1}(k, x)+r^{2}(p-q) H_{2}(k, x)-p^{2}(q-r) H_{3}(k, x)}{q^{2}(r-p)+r^{2}(p-q)+p^{2}(q-r)}\right]\right) \\
& =\frac{1}{\left|q^{2}(r-p)+r^{2}(p-q)+p^{2}(q-r)\right|} \\
& \quad \times \rho\left(\left(q^{2}(r-p)+r^{2}(p-q)+p^{2}(q-r) f(k, x)-q^{2}(r-p) H_{1}(k, x)\right.\right.
\end{aligned}
$$




$$
\left.-r^{2}(p-q) H_{2}(k, x)+p^{2}(q-r) H_{3}(k, x)\right)
$$

For convince, we assume that

$$
\begin{gathered}
\quad \frac{1}{\left|q^{2}(r-p)+r^{2}(p-q)+p^{2}(q-r)\right|}=\frac{1}{|A|} \\
\leq \frac{1}{|A|} \rho\left[\left(q^{2}(r-p) f(k, x)-q^{2}\left(r^{2}-p^{2}\right) f(k, x-1)\right.\right. \\
\left.+q^{2}(r-p) p r f(k, x-2)-q^{2}(r-p) H_{1}(k, x)\right) \\
+\left(r^{2}(p-q) f(k, x)-r^{2}\left(p^{2}-q^{2}\right) f(k, x-1)+r^{2}(p-q) q p f(k, x-2)\right. \\
+\left(p^{2}(q-r) f(k, x)-p^{2}\left(q^{2}-r^{2}\right) f(k, x-1)+p^{2}(q-r) q r f(k, x-2)\right. \\
\left.\left.\quad-p^{2}(q-r) H_{3}(k, x) \mid\right)\right] \\
\leq \frac{1}{|A|}\left[\frac{1}{1-|q|}+\frac{1}{1-|q|}+\frac{\left|q^{2}\right|}{1-\left|q^{2}\right|}\right] \epsilon \\
=\frac{1}{|A|}\left[\frac{2}{1-|q|}+\frac{\left|q^{2}\right|}{1-\left|q^{2}\right|}\right] \epsilon \\
=\frac{1}{|A|}\left[\frac{2(1+|q|)+|q|^{2}}{1-\left|q^{2}\right|}\right] \epsilon
\end{gathered}
$$

Putting the value of $|A|$ from we get the required result.

Hence,

$$
H(k, x)=\frac{q^{2}(r-p) H_{1}(k, x)+r^{2}(p-q) H_{2}(k, x)-p^{2}(q-r) H_{3}(k, x)}{q^{2}(r-p)+r^{2}(p-q)+p^{2}(q-r)}
$$

for all $x \in R$. It is easy to show that $H$ is a $k$-Tribonacci function satisfying .

\section{References}

[1] A. H. Sales, About $k$-Fibonacci numbers and their associated numbers, Int. J. of Math Forum, 6 (50) (2011), 2473-2479.

[2] Ashish, R. Chugh, Hyers-Ulam-Rassias stability of orthogonality cubic and quadratic functional equations, Int. J. of Pure and Applied Mathematics, 81 (1) (2012), 9-20. 
[3] Ashish, R. Chugh, Manoj Kumar, Hyers-Ulam-Rassias stability of functional equations in multi-Banch space, Int. J. of Pure and Applied Mathematics, 86 (4) (2013), 621-631.

[4] C. Park, S. Y. Jang, R. Saadati, Fuzzy approximate of homomorphisms, J. Comp. Anal. Appl., 14 (5) (2012), 833-841.

[5] D. H. Hyers, On the stability if linear functional equation, Proc. Natl. Acad. Sci. USA., 27 (1941), 221-224.

[6] D. H. Hyers, G. Isac, Th. M Rassias, Stability of Functional Equations in Several Variables, Birkhauser, Boston (1998).

[7] D. H. Hyers, Th. M. Rassias, Approximate homomorphisms, Aequationes Math., 44 (1992), 125-153.

[8] G. L. Forti, Hyers-Ulam stability of functional equations in several variables, Aequationes Math., 50 (1995), 143-190.

[9] G. Z. Eskandani, On the Hyers-Ulam-Rassias stability of an additive functional equation in quasi-Banach spaces, J. Math. Anal. Appl., 345 (2008), 405-409.

[10] G. Z. Eskandani, Th. M proper JCQ*triples, Mediterr. J. Math., 10 (2013), 1391-1400.

[11] G. Z. Zamani, H. Vaezi, Fuzzy approximation of an additive functional equation, J. Funct. Spaces Appl., 9 (2) (2011), 205-215.

[12] Iz. El-Fassi, S. Kabbaj, Hyers-Ulam-Rassias stability of orthogonal quadratic functional equations in modular spaces, ., 26 (1) (2015), 61-75.

[13] J. M. Rassias, On approximately. Rassias: Hyers-Ulam-Rassias stability of derivations in of approximately linear mappings by linear mappings, $J$. Functional Anal. USA, 46 (1982), 126-130.

[14] J. Musielak, Orlicz spaces and modular spaces, Lecture Notes in Math., Springer-Verlag, Berlin, (1034) (1983).

[15] K. Jun, Y. Lee, On the Hyers-Ulam-Rassias stability of a Pexiderized quadratic inequality, Math. Inequal. Appl., 4 (2001), 93-118.

[16] M. Bidkham, M. Hosseini, Hyers-Ulam stability of $k$-Fibonacci functional equation, Int. J. Nonlinear Anal. Appl., 2 (2011), 42-49. 
[17] M. Bidkham, M. Hosseini, C. Park, M. Eshaghi Gordji, Nearly $(k, s)$ Fibonacci functional equations in $\beta$-normed spaces, Aequationes Math., 83 (2012), 131-141.

[18] M. Kumar, Ashish, R. Chugh, The stability of cubic functional equations in 2-Banach space, Applied Mathematical Sciences, 7 (60) (2013), 2967-2977.

[19] M. Kumar, Renu Chugh, Ashish, Hyers-Ulam-Rassias stability of quadratic functional equations in 2-Banach spaces, Int. J. of Comp. Appl., 63 (8) (2013), 1-4.

[20] M. Kumar, Ashish, Stability of functional equations in multi-Banach space via fixed point approach, Int. J. of Comp. Appl., 44 (7) (2012), 35-40.

[21] M. Eshaghi Gordji, A. Divandari, C. Park, D. Y. Shin, Hyers-Ulam stability of a Tribonacci functional equation in 2-normed space, J. Comp. Anal. and Appl., 6 (3) (2014), 503-508.

[22] M. Gordji, M. Naderi, Th. M. Rassias: Solution and stability of tribonacci functional equation in non-archinedean Banach spaces, ?, (2011), 67-74.

[23] M. N. Parizi, M. E. Gordji, Hyers-Ulam stability of functional equations in modular functional spaces, J. of Math. and Comp. Sci., 10 (2014), 1-6.

[24] M. S. Moslehian, Th. M. Rassias: Stability of functional equation in NonArchimedean spaces, Applicable Analysis and Discrete Mathe., 1 (2007), 325-334.

[25] P. Gavruta, A generalization of the Hyers-Ulam-Rassias stability of approximately additive mappings, J. Math. Anal. Appl., 184 (1994), 431-436.

[26] P. Gavruta, L. Gavruta, A new method for the generalized Hyers-UlamRassias stability, Int. J. Nonlinear Anal. Appl., 1 (2010), 11-18.

[27] R. Ger and P. Semrl, The stability of exponential equation, Proc. Amer. Math. Soc., 124 (1996), 779-787.

[28] R. Chugh, Ashish, On the stability of functional equations in Random Normed spaces, J. Comp. Anal. Appl., 45 (11) (2012), 25-34.

[29] R. Chugh, Ashish, The stability of pexiderized cauchy functional equation, Int. J. of Pure and Applied Mathematics, 77 (5) (2012), 649-666. 
[30] R. Chugh and Ashish, On the stability of generalized Cauchy linear functional equation, Int. J. of Mathematical Analysis, 6, No.29 (2012), 14031413.

[31] S. Czerwik, Functional Equations and Inequalities in Servel Variables, World Scientific, New Jersey, London, Singapore, Hong Kong (2002).

[32] S. Jung, Hyers-Ulam stability of Fibonacci functional equation, Bull. Iranian Math. Soc., 35 (2009), 217-227.

[33] S. Jung, Hyers-Ulam-Rassias stability of functional equations in nonlinear analysis, Springer Optimization and Its Applications, 48, Springer, New York (2011).

[34] S. M. Ulam, Problems in Modern Mathematics, Chapter VI, Science ed., Wiley, New York (1940).

[35] T. Aoki, On the stability of linear transformation in Banach spaces, $J$. Math. Soc. Jpn., 2 (1950), 64-66.

[36] Th. M. Rassias, On the stability ofthe linear mapping in Banach spaces, Proc. Amer. Math. Soc., 72 (1978), 297-300. 